\title{
Evaluation of Antimicrobial Activity of ZITRITIDE, A Natural and Organic Antimicrobial Fogging Solution with Special Reference for Infection Prevention and Control in Hospital Environments and All Other Clean Room Facilities
}

\author{
Prangya Paramita Tripathy and Oliver Davis George*
}

Senior Microbiologist (Research, Development and Analysis), Old No. 73, New No. 2, S-Block, 18th Street, Anna Nagar, Chennai -600040, Tamil Nadu, India

*Corresponding author

\section{Ke y w o r d s \\ Bioflavonoid complex, Fogging, Fumigant, Antimicrobial, MRSA, Ecofriendly, Clean room, Citrus aurantium Amara extract, HAI (Hospital Acquired Infections). \\ Article Info \\ Accepted: 15 March 2017 Available Online: 10 April 2017}

\section{A B S T R A C T}

The aim of the present study was to evaluate the antimicrobial efficacy of a natural and organic fogging solution, ZITRITIDE where Citrus aurantium Amara extract (Bioflavonoid Complex) acts as an active ingredient. The antimicrobial efficacy of the formulation at two different concentrations $(0.2 \%$ and $0.5 \%)$ was assessed against eighteen bacteria, and four fungi prevalent in hospital and industrial environment. Both the concentration worked efficiently on gram positive, gram negative, spore forming anaerobic, and spore forming aerobic microorganisms. At $0.2 \%$ concentration Mycobacterium tuberculosis and Methicillin Resistant Staphylococcus aureus (MRSA) showed $2.43 \log$ reduction and $2.05 \mathrm{log}$ reduction respectively. Eleven bacteria of the group demonstrated $4 \log$ reductions (99.99\% killing efficiency) and 3 log reductions were achieved by four bacteria. At $0.5 \%$ product concentration 10 bacteria showed $99.99 \%$ killing efficiency with $4 \log$ reduction value. The killing efficiency of the product for MRSA and Mycobacterium tuberculosis is $99 \%$ i.e. $2 \log$ reduction at $0.5 \%$. Antifungal activity at $0.2 \%$ and $0.5 \%$ concentration was highest for Aspergillus flavus with efficiency percent of 99.9999 i.e. 6 log reduction followed by Aspergillus niger (99.999), Penicillium species (99.99\%) and Candida albicans (99.9\%). Application of ZITRITIDE as a fumigant in clean room areas revealed $98 \%-100 \%$ reduction in bacterial count and $86 \%-100 \%$ reduction in fungal count in controlled areas and $80 \%-95 \%$ reduction in bacterial count and $89 \%-100 \%$ fungal count in uncontrolled areas. The ZITRITIDE was also fogged in hospital environment and found to be effective. The results demonstrated ZITRITIDE is quite effective in controlling hospital acquired infections (HAI). Being nontoxic and ecofriendly nature of the active ingredients, the advantage of ZITRITIDE over other chemical fumigant was that it can be fogged in the presence of personnel working in clean room areas and also in the presence of doctors, nurses, other clinical, non clinical professionals, patients, attendants, visitors and supporting staffs in hospitals.

\section{Introduction}

The present study focused on a $100 \%$ natural and organic antimicrobial solution for fogging/fumigating in all types of healthcare facilities and clean room environments.
The active ingredient in the product is derived from the peels of Citrus aurantium Amara (Bitter orange) extract (Bioflavonoid Complex). The potential for inadvertent exposure of chemical fumigant to people and 
damage to surface or equipment is well known but due to lack of a suitable natural alternative, chemical fumigants are used worldwide. Thus the present study originated from the idea to minimize/eliminate the use of toxic chemical fumigants in all healthcare facilities and clean room environments. The product has been developed pro-actively to eliminate the incidences of microbial diseases which have become immune to chemical based alternatives in hospitals and many other public environments. The intended application is with special reference to infection prevention and control in hospital environments that consists of high risk to low risk areas and other healthcare facilities under various settings including Intensive care units (Neonatal ICU, Pediatric ICU, ICU's Cardio Thoracic Vascular Surgery, Respiratory infections (H1N1 units), Operation Theatres, Dialysis Unit, Burns Unit, Transfusion services unit, Central Sterile Services Department, Patient wards, Out Patient departments and so on.

The local name of the Citrus aurantium subsp. Amara is bitter orange and belongs to the family Rutaceae. It is a spiny evergreen tree and locally available in India. The common name in India is Narangi. Citrus plant is native to tropical Asia but it is also found in all tropical and subtropical countries. Phenethylamine alkaloids, octopamine, synephrine, tyramine, N-methyltyramine and hordenine are the most important bioactive constituents of Citrus aurantium fruits. In addition it is also rich in volatile oil, vitamin $\mathrm{C}$, and flavonoids (hesperidine, naringin) and having beneficial effects on human health (Pellati et al., 2002). Because of its increased use in various chronic and acute diseases, Citrus aurantium attains more research attention. Other uses include the uses of Citrus aurantium essential oil in foods, perfumes and also used in herbal medicines as a stimulant and appetite suppressant. In traditional Chinese medicine, it is used to treat nausea, indigestion, constipation, cancer, and cardiovascular effect.

C.aurantium essential oil contains linalool and limonenes (fragrant substance) that have antianxiety and sedative effects (CarvalhoFreitas et al., 2002). AntidepressantSynephrine-rich Citrus aurantium extracts have antidepressant effects (Song et al., 1996). The whole $C$. aurantium peel contains citral, limonene, and several citrus bioflavonoid, including hesperidin, neohesperidin, naringin, and rutin. Evidence suggests that these substances also have antiviral effect (Song et al., 1996).

The best method to kill or inactivate a micro organism, such as bacteria, fungi or a virus before it reaches a human cell is by using an effective antimicrobial agent, that is non-toxic to humans and animals, but toxic to any or all micro-organisms. Fogging is a sterilization technique that uses a special machine to create a mist which eliminates all pathogens, even ones that cannot be reached by conventional cleaning. Chemical based fogging solution generates many health related problems to the people/staff involved in fogging activity. Being so small, these particles remain suspended in the air as aerosols for long time and thus able to kill any airborne microbial contamination that they come to contact with. Gradually these particles settle onto all surfaces, even the areas never touched by conventional cleaning. Fogging solution continues to work and kill any surface contamination. Also there is a possibility that after few hours of fumigation, these aerosols remain in the air and inhaled by patients, staffs, doctors and all personnel involved in the room unknowingly. This may create a health problem in the long run if the solution is chemical based. Residual effect of chemical fogging solution is harmful. One of the major concerns of the chemical based 
solution is the generation of resistant strains of microorganisms which are often fatal to the society. Examples include Methicillin Resistant Staphylococcus aureus, Vancomycin Resistant Enterococus faecium, and Gentamicin Resistant Gram Negative Bacteria that are always associated with Hospital Acquired Infection. But if the solution is in natural and organic form and at the same time it is good enough to destroy pathogenic microorganisms like chemical alternatives, then all the above concerns can be addressed with confidence.

Thus the main objective of this study is to analyze and report the antimicrobial efficacy of a natural and organic product where the active ingredient is derived from Citrus aurantium Amara extract (Bioflavonoid Complex) with the brand name of ZITRITIDE for application in hospital environments combating hospital acquired infections and other clean room facilities as well. Being nontoxic and eco-friendly nature of the active ingredients, the advantage of ZITRITIDE over other chemical fumigant is that it can be fogged in the presence of personnel working in clean room areas and also in the presence of doctors, nurses, other clinical, non clinical professionals, patients, attendants, visitors and supporting staffs in hospitals.

To our knowledge this is the first of its kind of natural and organic fogging solution where the antimicrobial efficacies against a broad spectrum of microorganisms are reported. In this study the antimicrobial efficacy of the ZITRITIDE (an antimicrobial fogging solution) has been evaluated against a broad spectrum of microorganisms that includes bacteria and fungi. In addition ZITRITIDE solution was evaluated practically in a clean room manufacturing environment and hospital during working hours.

\section{Materials and Methods}

Preparation of active ingredient for ZITRITIDE

ZITRITIDE is prepared from the super concentrated solution that is derived from the extracts of Bitter orange (Citrus aurantium) (bioflavonoid complex).

The active ingredients are polymethoxy flavonoids (PMFs), (nobiletin and tangeretin) found in rich quantities in the peel of citrus fruits. PMFs were extracted from the peels of Citrus aurantium to make Citrus aurantium Amara extract (CAE) (DamiánReyna et al., 2015).

\section{Preparation of dilution/concentration}

The super concentrate is diluted to $1 \%$ with active ingredient concentration of $20 \%$ volume/volume (v/v). This is marked as stock solution. The stock solution is further diluted to $0.2 \%(4 \% \mathrm{v} / \mathrm{v})$ and $0.5 \%(10 \% \mathrm{v} / \mathrm{v})$ respectively with demineralized water.

\section{Identification of microorganisms based on product application}

The microorganisms involved were identified based on the application of the product and listed out. Then the antimicrobial activity of each product concentration against each microorganism was assessed as per section 2.4 .

\section{Assessment of antimicrobial activity using a time-kill procedure}

The scope of this protocol is to measure the biocidal potential of a liquid antimicrobial formulation using a time-kill procedure (ASTM E2315). 


\section{Preparation of microbial culture}

All the bacteria were grown on nutrient broth/or specific broth media up to $24 \mathrm{~h}$ to $48 \mathrm{~h}$ at $37{ }^{\circ} \mathrm{C}$ depending upon the test conditions (Table 1). For initial bacterial count, a saline control test tube $(9 \mathrm{~mL})$ was spiked with $1 \mathrm{~mL}$ of bacterial culture and enumerated by pour plate technique in nutrient agar and/or specific media wherever required. Fungi were cultivated in different media and cultivation conditions (Table 1a).

For testing the fungal culture, a spore preparation from a saline wash was used. For testing the test product $9 \mathrm{~mL}$ of product were inoculated with $1 \mathrm{~mL}$ of each microbial culture separately, vortexed for $2 \mathrm{~min}$. immediately. Each tube was kept for the specified contact time. After specified contact time, $1 \mathrm{~mL}$ of sample mixture were taken and enumerated by pour plate technique. Further dilutions were made wherever necessary.

All the experiments were performed in duplicate. $\log _{10}$ values of each count were calculated and the difference from the initial $\log _{10}$ value was reported. Efficiency percent/percent difference was interpreted from table 2 .

\section{Application of zitritide for fumigation in clean room facility}

ZITRITIDE was used at ten different locations of the clean room facility. With 5\% ZITRITIDE the bacterial count and fungal count reduction was reported in table 9. In clean room area and controlled area the solution achieved 98\%-100\% reduction in bacterial count whereas reduction in fungal count was achieved in a range of $86 \%-100 \%$. In uncontrolled area $80 \%-95 \%$ reduction in bacterial count was achieved whereas $89 \%$ $100 \%$ reduction was observed in fungal count.

\section{Application of ZITRITIDE fogging solution in a clean room environment}

For practical application $50 \mathrm{~mL}$ of $1 \%$ ZITRITIDE was diluted with $950 \mathrm{~mL}$ of normal water to make it 5\% solution of ZITRITIDE. This was used at ten different locations of the clean room facilities. For fumigation Biostar ${ }^{\mathrm{TM}}$ ULV fogger machine was used as per manufacturer's instruction (Table 10).

\section{Fumigation experiment protocol}

Plates were prepared for settle plate exposure for fumigation requirement. Soyabean Casein Digest Agar for bacteria and Sabroaud Dextrose Agar media plates for fungi were prepared, marked and kept aside. Before fumigation (Pre-Fumigation) plates were exposed in different locations for 10 minutes. The fumigation was done at different locations as mentioned in table 9. After 20 minutes of fumigation (Post-fumigation) the Petri dishes were again exposed at different locations for 10 minutes. All the Petri dishes were collected and incubated at respective incubators $\left(37{ }^{\circ} \mathrm{C}\right.$ for 24 to 48 hour for bacteria and $25{ }^{\circ} \mathrm{C}$ for 5 to 7 days for fungi). All the results were recorded.

\section{Results and Discussion}

\section{Identification of microorganisms}

All possible sites of applications for the product were identified and the microorganisms' presence in the particular site was listed in tables 3 and 4. These microorganisms are generally prevalent in hospital and other environments. This includes high risk areas in hospitals under various settings including Respiratory infections (H1N1 units), Cardiothoracic surgery units, Intensive care units (Neonatal ICU, Pediatric ICU, ICU's), Vascular Surgery, 
Operation Theatres, Dialysis Unit, Burns Unit, Transfusion services unit, Central Sterile Services Department, Patient wards, outpatient departments and so on.

\section{Assessment of antimicrobial activity}

At $0.2 \%$ concentration the product showed highest $\log$ reduction (5.03) for Serratia marcescens. The concentration works efficiently on gram positive, gram negative, spore forming anaerobic, and spore forming aerobic microorganisms.

Mycobacterium tuberculosis and MRSA showed $2.43 \log$ reduction and $2.05 \log$ reduction respectively. $4 \log$ reductions (99.99\% killing efficiency) were achieved by 11 bacteria of the group whereas $3 \log$ reductions were achieved by four microorganisms (Table 5).

At $0.5 \%$ product concentration out of 18 bacteria, 10 bacteria showed $99.99 \%$ killing efficiency with $4 \log$ reduction value.

Five bacteria of the group demonstrated $3 \mathrm{log}$ reduction i.e. $99.9 \%$ killing efficiency. The killing efficiency of the product for MRSA and Mycobacterium tuberculosis is $99 \%$ i.e. 2 $\log$ reduction (Table 6).

At $0.2 \%$ concentration the antifungal activity was highest for Aspergillus flavus with efficiency percent of 99.9999 i.e. 6 log reduction followed by Aspergillus niger (99.999), Penicillium species (99.99\%) and Candida albicans (99.9\%) (Table 7).

At $0.5 \%$ concentration, the antifungal activity of ZITRITIDE is $5.3222 \log$ reduction followed by Aspergillus niger (5.3374), Penicillium species (99.99) and Candida albicans (99.9) (Table 8).

\section{Zitritide combating Hospital Acquired Infection (HAI)}

The prevalence of pathogens in hospitals which are usually involved in hospital based infections is taken into consideration in this study. $2 \log$ reduction (99\%) for MRSA in 10 min were observed whereas for Vancomycin Resistant Enterococcus faecium, E. coli, S. aureus, $P$. aeruginosa 4 log reduction $(99.99 \%)$ were observed. The product's applications on HAI associated with fungi were also studied. Aspergillus flavus showed highest i.e. $6 \log$ reduction in 5 minutes. Aspergillus niger is the next fungi in which the product application showed 5 log reductions. Upon Candida albicans $3 \mathrm{log}$ reduction was observed with a contact time period over 15 mins (Figure1 and Figure 2). At both the product concentration, the effect on bacteria and fungi are same.

\section{Application of zitritide for fumigation in hospital environments}

Preliminary tests and trials have been conducted by the Department of Microbiology, Trivendrum Medical College, and ZITRITIDE's favorable report has been obtained after testing of the effectiveness of the pathogenic microorganisms (Sarala Devi, Personal Communication).

ZITRITIDE being a water-based broad spectrum anti-microbial product with $100 \%$ natural and organic ingredient can be fogged to control the levels of environmental microorganisms. It does not require heating and does not use any kind of chemical solvents or compounds or substances. The active ingredient in ZITRITIDE is derived from the peels of Citrus aurantium Amara extract (bioflavonoid complex). 
Table.1 Media used for bacteria

\begin{tabular}{|c|c|c|c|}
\hline Sl. No & Name of the Microorganisms & Media Used & $\begin{array}{c}\text { Other } \\
\text { Media/Solution Used } \\
\text { for Analysis }\end{array}$ \\
\hline 1 & Acinetobacter species & $\begin{array}{c}\text { Leeds } \\
\text { Acinetobacter } \\
\text { Agar Base }\end{array}$ & \multirow{18}{*}{$\begin{array}{l}\text { Peptone Water, D/E } \\
\text { Neutralizing Broth, } \\
\text { Nutrient Agar, } \\
\text { Chloramphenicol } \\
\text { Yeast Dextrose agar, } \\
\text { Blood Agar }\end{array}$} \\
\hline 2 & Bacillus cereus & $\begin{array}{l}\text { AK Agar No. } 2 \\
\text { (Sporulating } \\
\text { Agar) }\end{array}$ & \\
\hline 3 & Clostridium perfringenes & $\begin{array}{c}\text { Anaerobic egg } \\
\text { agar Base }\end{array}$ & \\
\hline 4 & Clostridium sporogenes & $\begin{array}{c}\text { Anaerobic egg } \\
\text { agar Base }\end{array}$ & \\
\hline 5 & Coagulase Negative Staphylococci & Nutrient Agar & \\
\hline 6 & Enterococcus species & Nutrient Agar & \\
\hline 7 & Escherichia coli & EMB Agar & \\
\hline 8 & Klebsiella pneumoniae & $\begin{array}{l}\text { Mac Conkey } \\
\text { Agar }\end{array}$ & \\
\hline 9 & Methicillin Resistant Staphylococcus aureus & $\begin{array}{c}\text { Nutrient } \\
\text { Agar/Nutrient } \\
\text { Broth }\end{array}$ & \\
\hline 10 & Mycobacterium tuberculosis & $\begin{array}{l}\text { Lowenstein- } \\
\text { Jensen Medium }\end{array}$ & \\
\hline 11 & Proteus mirabilis & $\begin{array}{l}\text { Mac Conkey } \\
\text { Agar }\end{array}$ & \\
\hline 12 & Proteus vulgaris & $\begin{array}{l}\text { Mac Conkey } \\
\text { Agar }\end{array}$ & \\
\hline 13 & Pseudomonas aeruginosa & Cetrimide Agar & \\
\hline 14 & Salmonella cholreasuis & $\begin{array}{c}\text { Bismuth } \\
\text { sulphite Agar }\end{array}$ & \\
\hline 15 & Serratia marcescens & Nutrient Agar & \\
\hline 16 & Staphylococcus aureus & $\begin{array}{l}\text { Baird Parker } \\
\text { Agar }\end{array}$ & \\
\hline 17 & Streptococcus pyogenes & $\begin{array}{l}\text { Mannitol Salt } \\
\text { Agar }\end{array}$ & \\
\hline 18 & Vancomycin Resistant Enterococcus faecium & $\begin{array}{c}\text { Vancomycin } \\
\text { Resistant } \\
\text { Enterococci } \\
\text { (VRE) Agar }\end{array}$ & \\
\hline
\end{tabular}


Table.1a Media used for fungi

\begin{tabular}{|c|c|c|c|}
\hline S. No. & $\begin{array}{c}\text { Name of the } \\
\text { Microorganisms }\end{array}$ & Media used for Analysis & $\begin{array}{l}\text { Other Media/Solution Used } \\
\text { for Analysis }\end{array}$ \\
\hline 1 & Aspergillus flavus & Sabouraud Dextose Agar & \multirow{4}{*}{$\begin{array}{l}\text { Peptone Water, D/E } \\
\text { Neutralizing Broth }\end{array}$} \\
\hline 2 & Aspergillus niger & Sabouraud Dextose Agar & \\
\hline 3 & Candida albicans & $\begin{array}{l}\text { Corn Meal Agar/Potato } \\
\text { Dextrose Agar }\end{array}$ & \\
\hline 4 & Penicillium sp. & Sabouraud Dextose Agar & \\
\hline
\end{tabular}

Table.2 Efficiency percent for log reduction values

\begin{tabular}{|c|c|}
\hline Log Difference & Efficiency Percent/Percent Difference \\
\hline 1 Log Reduction & $90 \%$ Reduction \\
\hline 2 Log Reduction & $99 \%$ Reduction \\
\hline 3 Log Reduction & $99.9 \%$ Reduction \\
\hline 4 Log Reduction & $99.99 \%$ Reduction \\
\hline 5 Log Reduction & $99.999 \%$ Reduction \\
\hline 6 Log Reduction & $99.9999 \%$ Reduction \\
\hline
\end{tabular}

Table.3 Zitritide - list of fungi

\begin{tabular}{|c|l|c|}
\hline S. No. & \multicolumn{1}{|c|}{$\begin{array}{c}\text { Name of the } \\
\text { Microorganisms }\end{array}$} & $\begin{array}{c}\text { Applications } \\
1\end{array}$ \\
Aspergillus flavus & $\begin{array}{c}\text { Fomites of Operating theatres, Patient } \\
\text { rooms, Hospital Environments, } \\
\text { Healthcare centres }\end{array}$ \\
\hline 2 & Aspergillus niger & $\begin{array}{c}\text { Fomites of Operating theatres, Patient } \\
\text { rooms, Hospital Environments, } \\
\text { Healthcare centres }\end{array}$ \\
\hline 3 & Candida albicans & $\begin{array}{c}\text { Fomites of Operating theatres, Patient } \\
\text { rooms, Hospital Environments, } \\
\text { Healthcare centres }\end{array}$ \\
\hline 4 & Penicillium sp. & $\begin{array}{c}\text { Fomites of Operating theatres, Patient } \\
\text { rooms, Hospital Environments, } \\
\text { Healthcare centres }\end{array}$ \\
\hline
\end{tabular}


Table.4 Zitritide - list of bacteria

\begin{tabular}{|c|c|c|}
\hline Sl. No & Name of the Microorganisms & Applications \\
\hline 1 & Acinetobacter species & $\begin{array}{c}\text { Fomites of Operating theatres, Patient rooms, } \\
\text { Hospital Environments, Healthcare centres }\end{array}$ \\
\hline 2 & Bacillus cereus & $\begin{array}{l}\text { Fomites of Operating theatres, Patient rooms, } \\
\text { Hospital Environments, Healthcare centres }\end{array}$ \\
\hline 3 & Clostridium perfringenes & $\begin{array}{l}\text { Fomites of Operating theatres, Patient rooms, } \\
\text { Hospital Environments, Healthcare centres }\end{array}$ \\
\hline 4 & Clostridium sporogenes & $\begin{array}{l}\text { Fomites of Operating theatres, Patient rooms, } \\
\text { Hospital Environments, Healthcare centres }\end{array}$ \\
\hline 5 & $\begin{array}{l}\text { Coagulase Negative } \\
\text { Staphylococci }\end{array}$ & $\begin{array}{l}\text { Fomites of Operating theatres, Patient rooms, } \\
\text { Hospital Environments, Healthcare centres }\end{array}$ \\
\hline 6 & Enterococcus species & $\begin{array}{c}\text { Operating theatres, Patient rooms, Hospital } \\
\text { Environments, Healthcare centres }\end{array}$ \\
\hline 7 & Escherichia coli & $\begin{array}{l}\text { Fomites of Operating theatres, Patient rooms, } \\
\text { Hospital Environments, Healthcare centres }\end{array}$ \\
\hline 8 & Klebsiella pneumoniae & $\begin{array}{l}\text { Operating theatres, Patient rooms, Hospital } \\
\text { Environments, Healthcare centres }\end{array}$ \\
\hline 9 & $\begin{array}{c}\text { Methicillin Resistant } \\
\text { Staphylococcus aureus }\end{array}$ & $\begin{array}{l}\text { Operating theatres, Patient rooms, Hospital } \\
\text { Environments, Healthcare centres }\end{array}$ \\
\hline 10 & Mycobacterium tuberculosis & $\begin{array}{c}\text { Fomites of Operating theatres, Patient rooms, } \\
\text { Hospital Environments, Healthcare centres }\end{array}$ \\
\hline 11 & Proteus mirabilis & $\begin{array}{l}\text { Fomites of Operating theatres, Patient rooms, } \\
\text { Hospital Environments, Healthcare centres }\end{array}$ \\
\hline 12 & Proteus vulgaris & $\begin{array}{l}\text { Fomites of Operating theatres, Patient rooms, } \\
\text { Hospital Environments, Healthcare centres }\end{array}$ \\
\hline 13 & Pseudomonas aeruginosa & $\begin{array}{l}\text { Fomites of Operating theatres, Patient rooms, } \\
\text { Hospital Environments, Healthcare centres }\end{array}$ \\
\hline 14 & Salmonella cholreasuis & $\begin{array}{l}\text { Fomites of Operating theatres, Patient rooms, } \\
\text { Hospital Environments, Healthcare centres }\end{array}$ \\
\hline 15 & Serratia marcescens & $\begin{array}{l}\text { Fomites of Operating theatres, Patient rooms, } \\
\text { Hospital Environments, Healthcare centres }\end{array}$ \\
\hline 16 & Staphylococcus aureus & $\begin{array}{l}\text { Fomites of Operating theatres, Patient rooms, } \\
\text { Hospital Environments, Healthcare centres }\end{array}$ \\
\hline 17 & Streptococcus pyogenes & $\begin{array}{l}\text { Fomites of Operating theatres, Patient rooms, } \\
\text { Hospital Environments, Healthcare centres }\end{array}$ \\
\hline 18 & $\begin{array}{l}\text { Vancomycin Resistant } \\
\text { Enterococcus faecium }\end{array}$ & $\begin{array}{l}\text { Fomites of Operating theatres, Patient rooms, } \\
\text { Hospital Environments, Healthcare centres }\end{array}$ \\
\hline
\end{tabular}


Table.5 Assessment of antibacterial activity of ZITRITIDE @ $0.2 \%$ concentration

\begin{tabular}{|c|c|c|c|c|c|c|}
\hline Sl. No & Name of the Bacteria & $\begin{array}{c}\text { Initial } \\
\text { Log }_{10} \\
\text { Count }\end{array}$ & $\begin{array}{l}\text { Contact } \\
\text { Time }\end{array}$ & $\begin{array}{l}\text { Final } \\
\text { Log }_{10} \\
\text { Count }\end{array}$ & $\begin{array}{c}\log _{10} \\
\text { Reduction } \\
\text { Count }\end{array}$ & $\begin{array}{l}\text { Efficiency } \\
\text { percent } \\
(\%)\end{array}$ \\
\hline 1 & $\begin{array}{c}\text { Acinetobacter species NCIM } \\
2886\end{array}$ & 6.415 & 5 Min & 2.0414 & 4.3736 & 99.99 \\
\hline 2 & Bacillus cereus NCIM 2156 & 5.875 & $15 \mathrm{Min}$ & 2.857 & 3.018 & 99.9 \\
\hline 3 & $\begin{array}{c}\text { Clostridium perfringenes } \\
\text { NCIM } 2677\end{array}$ & 5.9868 & $10 \mathrm{Min}$ & 2.6232 & 3.3636 & 99.9 \\
\hline 4 & $\begin{array}{c}\text { Clostridium sporogenes } \\
\text { NCIM } 2559\end{array}$ & 6.0414 & $10 \mathrm{Min}$ & 2.6128 & 3.4286 & 99.9 \\
\hline 5 & $\begin{array}{c}\text { Coagulase negative } \\
\text { Staphylococci } \text { MTCC } 8924\end{array}$ & 6.813 & $10 \mathrm{Min}$ & 2.176 & 4.637 & 99.99 \\
\hline 6 & $\begin{array}{c}\text { Enterococcus species } \\
\text { NCIM } 5253\end{array}$ & 6.531 & $30 \mathrm{Min}$ & 3.279 & 3.252 & 99.9 \\
\hline 7 & $\begin{array}{c}\text { Escherichia coli } \\
\text { NCIM } 2065\end{array}$ & 6.8751 & 5 Min & 2.6128 & 4.2623 & 99.99 \\
\hline 8 & $\begin{array}{l}\text { Klebsiella pneumoniae } \\
\text { NCIM } 2957\end{array}$ & 7.447 & 5 Min & 2.949 & 4.498 & 99.99 \\
\hline 9 & $\begin{array}{c}\text { Methicillin Resistant } \\
\text { Staphylococcus aureus } \\
\text { MTCC } 3610\end{array}$ & 5.978 & $10 \mathrm{Min}$ & 3.924 & 2.053 & 99 \\
\hline 10 & $\begin{array}{c}\text { Mycobacterium tuberculosis } \\
\text { MTCC } 300\end{array}$ & 5.732 & 5 Min & 3.301 & 2.431 & 99 \\
\hline 11 & $\begin{array}{l}\text { Proteus mirabilis } \\
\text { NCIM } 5296 \\
\end{array}$ & 6.7324 & $10 \mathrm{Min}$ & 2.3979 & 4.3345 & 99.99 \\
\hline 12 & $\begin{array}{c}\text { Proteus vulgaris } \\
\text { NCIM } 2027 \\
\end{array}$ & 6.4914 & $10 \mathrm{Min}$ & 1.8451 & 4.6463 & 99.99 \\
\hline 13 & $\begin{array}{l}\text { Pseudomonas aeruginosa } \\
\text { NCIM 5029, ATCC } 27853\end{array}$ & 7.643 & $10 \mathrm{Min}$ & 3.491 & 4.152 & 99.99 \\
\hline 14 & $\begin{array}{l}\text { Salmonella enterica } \\
\text { NCIM5256 }\end{array}$ & 6.5563 & $2 \mathrm{Min}$ & 1.6532 & 4.9031 & 99.99 \\
\hline 15 & $\begin{array}{c}\text { Serratia marcescens } \\
\text { NCIM } 2919 \\
\end{array}$ & 6.7324 & $10 \mathrm{Min}$ & 1.699 & 5.0334 & 99.999 \\
\hline 16 & $\begin{array}{c}\text { Staphylococcus aureus } \\
\text { NCIM 5345, ATCC } 6538\end{array}$ & 8.079 & $15 \mathrm{Min}$ & 3.477 & 4.602 & 99.99 \\
\hline 17 & $\begin{array}{c}\text { Streptococcus pyogenes } \\
\text { NCIM } 2608\end{array}$ & 7.380 & 10 Min & 2.881 & 4.499 & 99.99 \\
\hline 18 & $\begin{array}{c}\text { Vancomycin Resistant } \\
\text { Enterococcus faecium } \\
\text { NCIM } 5366\end{array}$ & 6.806 & 10 Min & 2.788 & 4.028 & 99.99 \\
\hline
\end{tabular}


Table.6 Assessment of antimicrobial activity of ZITRITIDE @ $0.5 \%$ concentration

\begin{tabular}{|c|c|c|c|c|c|c|}
\hline Sl. No & Name of the Bacteria & $\begin{array}{l}\text { Initial } \\
\text { Log10 } \\
\text { Count }\end{array}$ & $\begin{array}{l}\text { Contact } \\
\text { Time }\end{array}$ & $\begin{array}{l}\text { Final } \\
\text { Log }_{10} \\
\text { Count }\end{array}$ & $\begin{array}{c}\log _{10} \\
\text { Reduction } \\
\text { Count }\end{array}$ & $\begin{array}{l}\text { Efficiency } \\
\text { percent } \\
(\%)\end{array}$ \\
\hline 1 & $\begin{array}{c}\text { Acinetobacter species NCIM } \\
2886\end{array}$ & 6.415 & 10 Min & 1.6532 & 4.7618 & 99.99 \\
\hline 2 & $\begin{array}{l}\text { Bacillus cereus } \\
\text { NCIM } 2156\end{array}$ & 5.875 & 15 Min & 2.756 & 3.119 & 99.9 \\
\hline 3 & $\begin{array}{c}\text { Clostridium perfringenes } \\
\text { NCIM } 2677\end{array}$ & 5.9868 & 5 Min & 2.3424 & 3.6444 & 99.9 \\
\hline 4 & $\begin{array}{c}\text { Clostridium sporogenes } \\
\text { NCIM } 2559\end{array}$ & 6.0414 & 30 Min & 2.6435 & 3.3979 & 99.9 \\
\hline 5 & $\begin{array}{c}\text { Coagulase negative } \\
\text { Staphylococci } \\
\text { MTCC 8924 } \\
\end{array}$ & 6.813 & 10 Min & 2.041 & 4.772 & 99.99 \\
\hline 6 & Enterococcus species NCIM 5253 & 6.531 & 30 Min & 3.362 & 3.169 & 99.9 \\
\hline 7 & $\begin{array}{c}\text { Escherichia coli } \\
\text { NCIM } 2065\end{array}$ & 6.8751 & 5 Min & 2.2553 & 4.6198 & 99.99 \\
\hline 8 & $\begin{array}{c}\text { Klebsiella pneumoniae NCIM } \\
2957\end{array}$ & 7.447 & 5 Min & 2.699 & 4.748 & 99.99 \\
\hline 9 & $\begin{array}{c}\text { Methicillin Resistant } \\
\text { Staphylococcus aureus MTCC } \\
\mathbf{3 6 1 0} \\
\end{array}$ & 5.978 & 10 Min & 3.785 & 2.192 & 99 \\
\hline 10 & $\begin{array}{c}\text { Mycobacterium tuberculosis } \\
\text { MTCC300 }\end{array}$ & 5.732 & 5 Min & 2.820 & 2.913 & 99 \\
\hline 11 & $\begin{array}{c}\text { Proteus mirabilis } \\
\text { NCIM } 5296 \\
\end{array}$ & 6.7324 & 10 Min & 2.000 & 4.7324 & 99.99 \\
\hline 12 & $\begin{array}{c}\text { Proteus vulgaris } \\
\text { NCIM } 2027 \\
\end{array}$ & 6.4914 & 5 Min & 1.6628 & 4.8286 & 99.99 \\
\hline 13 & $\begin{array}{l}\text { Pseudomonas aeruginosa } \\
\text { NCIM 5029, ATCC } 27853\end{array}$ & 7.643 & 10 Min & 3.079 & 4.564 & 99.99 \\
\hline 14 & Salmonella enterica NCIM 5256 & 6.5563 & 2 Min & 1.6532 & 4.931 & 99.99 \\
\hline 15 & $\begin{array}{c}\text { Serratia marcescens } \\
\text { NCIM } 2919\end{array}$ & 6.7324 & 30 Min & 1.6021 & 5.1303 & 99.999 \\
\hline 16 & $\begin{array}{c}\text { Staphylococcus aureus } \\
\text { NCIM 5345, } \\
\text { ATCC } 6538 \\
\end{array}$ & 8.079 & 10 Min & 3.477 & 4.602 & 99.99 \\
\hline 17 & $\begin{array}{c}\text { Streptococcus pyogenes NCIM } \\
2608 \\
\end{array}$ & 7.380 & 10 Min & 2.845 & 4.535 & 99.99 \\
\hline 18 & $\begin{array}{c}\text { Vancomycin Resistant } \\
\text { Enterococcus faecium NCIM } \\
5366\end{array}$ & 6.806 & 10 Min & 2.903 & 3.903 & 99.9 \\
\hline \multicolumn{7}{|c|}{ Results are expressed as average values of two repeated experiment. } \\
\hline
\end{tabular}


Table.7 Assessment of Antifungal Activity of ZITRITIDE @ 0.2\% concentration

\begin{tabular}{|c|c|c|c|c|c|c|}
\hline Sl. No & $\begin{array}{c}\text { Name of the } \\
\text { Fungi }\end{array}$ & $\begin{array}{c}\text { Initial } \\
\text { Log }_{10} \\
\text { Count }\end{array}$ & $\begin{array}{l}\text { Contact } \\
\text { Time }\end{array}$ & $\begin{array}{l}\text { Final } \\
\text { Log10 } \\
\text { Count }\end{array}$ & $\begin{array}{c}\log _{10} \\
\text { Reduction } \\
\text { Count }\end{array}$ & $\begin{array}{c}\text { Efficiency } \\
\text { percent } \\
(\%)\end{array}$ \\
\hline 1 & $\begin{array}{c}\text { Aspergillus } \\
\text { flavus NCIM } \\
1316\end{array}$ & 6.7993 & $10 \mathrm{Min}$ & 1.0000 & 5.7993 & 99.9999 \\
\hline 2 & $\begin{array}{l}\text { Aspergillus } \\
\text { niger } \\
\text { NCIM1317 }\end{array}$ & 6.9395 & 5 Min & 1.6021 & 5.3374 & 99.999 \\
\hline 3 & $\begin{array}{c}\text { Penicillium } \\
\text { species } \\
\text { NCIM } 1108\end{array}$ & 6.1461 & $2 \mathrm{Min}$ & 1.6532 & 4.4929 & 99.99 \\
\hline 4 & $\begin{array}{c}\text { Candida } \\
\text { albicans } \\
\text { NCIM } 3100\end{array}$ & 5.5315 & $15 \mathrm{Min}$ & 2.3222 & 3.2093 & 99.9 \\
\hline
\end{tabular}

Table.8 Assessment of antifungal activity for ZITRITIDE @ $0.5 \%$ concentration

\begin{tabular}{|c|l|c|c|c|c|c|}
\hline SI. No & \multicolumn{1}{|c|}{$\begin{array}{c}\text { Name of the } \\
\text { Fungi }\end{array}$} & $\begin{array}{c}\text { Initial } \\
\mathbf{L o g}_{\mathbf{1 0}} \\
\text { Count }\end{array}$ & $\begin{array}{c}\text { Contact } \\
\text { Time }\end{array}$ & $\begin{array}{c}\text { Final } \\
\mathbf{L o g}_{10} \\
\text { Count }\end{array}$ & $\begin{array}{c}\text { Log }_{10} \\
\text { Reduction } \\
\text { Count }\end{array}$ & $\begin{array}{c}\text { Efficiency } \\
\text { percent } \\
(\%)\end{array}$ \\
\hline 1 & $\begin{array}{l}\text { Aspergillus flavus } \\
\text { NCIM 1316 }\end{array}$ & 6.7993 & 5 Min & 1.4771 & 5.3222 & 99.9999 \\
\hline 2 & $\begin{array}{l}\text { Aspergillus niger } \\
\text { NCIM 1317 }\end{array}$ & 6.9395 & 5 Min & 1.6021 & 5.3374 & 99.999 \\
\hline $\begin{array}{l}\text { Penicillium } \\
\text { species } \\
\text { NCIM 1108 }\end{array}$ & 6.1461 & 2 Min & 1.4771 & 4.6690 & 99.99 \\
\hline 4 & $\begin{array}{l}\text { Candida albicans } \\
\text { NCIM 3100 }\end{array}$ & 5.5315 & 15 Min & 2.3222 & 3.2093 & 99.9 \\
\hline Results are expressed as average values of two repeated experiment. & & \\
\hline
\end{tabular}


Table.9 Application of ZITRITIDE fogging solution in clean room facility

\begin{tabular}{|c|c|c|c|c|c|c|c|c|}
\hline & & & Bacteri & $\begin{array}{l}\text { l Colony Cor } \\
\text { Cfu/Plate) }\end{array}$ & nts (In & Fungal & Count (In Cf & (Plate) \\
\hline $\begin{array}{l}\text { Sl. } \\
\text { No }\end{array}$ & $\begin{array}{c}\text { Area of } \\
\text { Fumigation }\end{array}$ & $\begin{array}{l}\text { Labelled } \\
\text { as }\end{array}$ & $\begin{array}{c}\text { Before } \\
\text { Fumigation }\end{array}$ & $\begin{array}{c}\text { After } \\
\text { Fumigation }\end{array}$ & $\begin{array}{c}\text { Percent } \\
\text { Reduction } \\
(\%)\end{array}$ & $\begin{array}{c}\text { Before } \\
\text { Fumigation }\end{array}$ & $\begin{array}{c}\text { After } \\
\text { Fumigation }\end{array}$ & $\begin{array}{c}\text { Percent } \\
\text { Reduction } \\
(\%)\end{array}$ \\
\hline 1 & $\begin{array}{c}\text { Biosafety } \\
\text { Room (BS- } \\
\text { MB) }\end{array}$ & $\begin{array}{l}\text { (BS- } \\
\mathrm{MB})\end{array}$ & 6 & $<1 *$ & 100 & 3 & $<1 *$ & 100 \\
\hline 2 & Food Hall & $\mathrm{FH}$ & 45 & $<1^{*}$ & 100 & 8 & 1 & 88 \\
\hline 3 & $\begin{array}{l}\text { Primary } \\
\text { Packing } \\
\end{array}$ & $\mathrm{PP}$ & 5 & $<1 *$ & 100 & 15 & $<1 *$ & 100 \\
\hline 4 & $\begin{array}{l}\text { Quarantine } \\
\text { Store, } 43 F\end{array}$ & QS & 13 & $<1 *$ & 100 & 1 & $<1 *$ & 100 \\
\hline 5 & $\begin{array}{c}\text { Men's } \\
\text { Washroom }\end{array}$ & MWR & 207 & 11 & 95 & 7 & 1 & 86 \\
\hline 6 & $\begin{array}{c}\text { Women's } \\
\text { Washroom }\end{array}$ & WWR & 55 & 10 & 82 & $\begin{array}{c}\text { TNTC }(> \\
300)\end{array}$ & 3 & 99 \\
\hline 7 & $\begin{array}{c}\text { Laser } \\
\text { marking }\end{array}$ & LM & 123 & 2 & 98 & 6 & 1 & 100 \\
\hline 8 & $\begin{array}{c}\text { Production } \\
\text { Area }\end{array}$ & PA & 20 & 4 & 80 & 7 & $<1 *$ & 100 \\
\hline 9 & $\begin{array}{l}\text { Pass Box, } \\
\text { Production } \\
\text { Area }\end{array}$ & PB1-PA & 11 & $<1 *$ & 100 & 3 & $<1 *$ & 100 \\
\hline 10 & $\begin{array}{l}\text { Humidity } \\
\text { Chamber } \\
\text { Room } \\
\text { (Stability } \\
\text { Room) }\end{array}$ & SR-MB & 5 & $<1 *$ & 100 & 9 & 1 & 89 \\
\hline $\begin{array}{l}\text { Re } \\
* \mathbf{N} \\
\mathbf{T N}\end{array}$ & $\begin{array}{l}\text { are expresse } \\
\text { icrobial grow } \\
\text { Too Numero }\end{array}$ & $\begin{array}{l}\text { Is average } \\
\text { observed } \\
\text { to Count }\end{array}$ & ues of two $r$ & ed experime & & & & \\
\hline
\end{tabular}


Table.10 Manufacturer's instruction table for ULV fogger machine

\begin{tabular}{|c|c|c|c|}
\hline Sl. No. & Room Sizes (m3/feet3) & Total Volume & Duration \\
\hline 1 & $30 / 1000$ & $400 \mathrm{~mL}$ & 5 mins \\
\hline 2 & $60 / 2000$ & $800 \mathrm{~mL}$ & 10 mins \\
\hline 3 & $90 / 3000$ & $1200 \mathrm{~mL}$ & 15 mins \\
\hline 4 & $120 / 4000$ & $1600 \mathrm{~mL}$ & 20 mins \\
\hline 5 & $150 / 5000$ & $2000 \mathrm{~mL}$ & 25 Mins \\
\hline 6 & $180 / 6000$ & $2400 \mathrm{~mL}$ & 30 mins \\
\hline
\end{tabular}

Figure I: Percent Efficiency of 0.2\% ZITRITIDE for Microorganisms involved in Hospital Aquired Infections

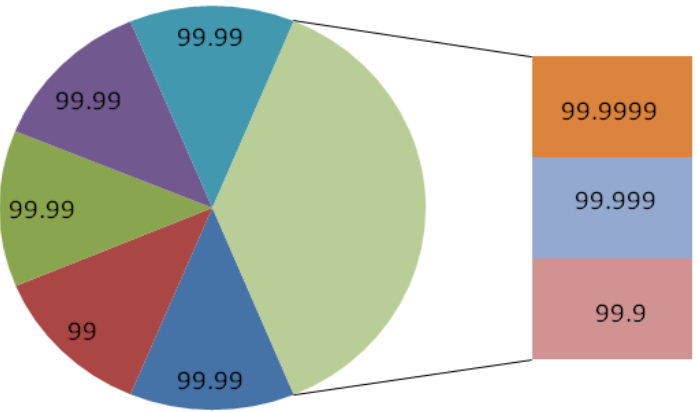

1 Escherichiacoli NCIM 20655 Min

- 2 Methicillin Resistant Staphylococcus aureus MTCC 3610 IOMin

- 3 Pseudomonas aeruginosa NCIM 5029, ATCC $2785310 \mathrm{Min}$

- 4 Staphylococcus aureus NCIM 5345, ATCC $653815 \mathrm{Min}$

- 5 Vancomycin Resistant Enterococcus faecium NCIM 5366 $10 \mathrm{Min}$

- 6 Aspergillus flavus NCIM 131610 Min

" 7 Aspergillus niger NCIM13175 Min

- 8 Candidaalbicans NCIM 310015 Min 


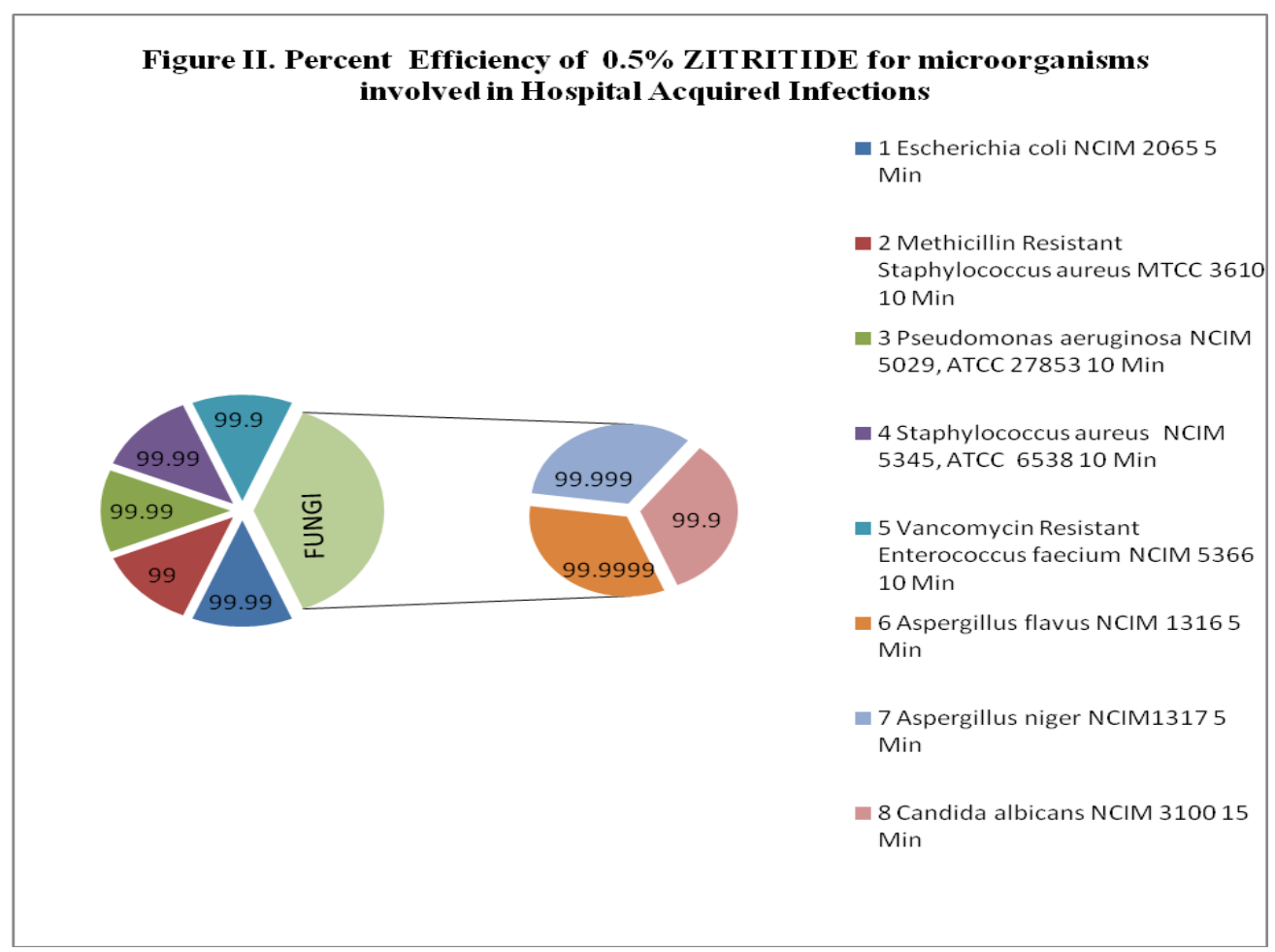

This study evaluated the Novel application of a natural and organic product (ZITRITIDE) derived from the above formulation as a fumigant/fogging solution in hospital environments. Infection control in any hospital or health care setting/facility is a very vital and important discipline concerned with preventing/controlling nosocomial and other healthcare associated infections/cross contaminations, to the patients, attendants, visitors, doctors, nurses and all other technical and non-technical support staff who practically come in contact with any or all such risks. The main advantage of ZITRITIDE over other chemical fumigants is that it was applied in the presence of doctors, nurses, other clinical, non clinical professionals, patients, attendants, visitors and support staffs and found to be effective (Sarala devi, Personal Communication).

As discussed in the results section the product acts efficiently with 18 bacteria and 4 fungi respectively prevalent in critical and high risk hospital departments. The product also works well for HAI pathogens at $0.2 \%$ and $0.5 \%$ concentration.
In a study Citrus limonum and Citrus aurantium essential oils (EOs) compared to $0.2 \%$ chlorohexidine (CHX) and $1 \%$ sodium hypochlorite $(\mathrm{NaOCl})$ on multispecies biofilms formed by Candida albicans, Enterococcus faecalis and Escherichia coli. C. aurantium EO and $\mathrm{NaOCl}$ inhibited the growth of all microorganisms in multi-species biofilms. $100 \%$ reduction of $C$. albicans and E. coli, and $49.3 \%$ reduction of $E$. faecalis were observed with $C$. limonum EO. A reduction of $68.8 \%$ of C. albicans and $86.7 \%$ of E. coli was observed in case of $\mathrm{CHX}$ and found to be less effective. Oliveria et al., (2014) was observed the EOs was effective in controlling multi-species biofilms, and the antimicrobial activity of EOs was higher than those of $\mathrm{CHX}$ and $\mathrm{NaOCl}$. Usually the peels of the citrus fruits are considered to be the waste product of citrus processing industries. In the present study, the peel extracts of Citrus aurantium Amara were used as active ingredient in the product that exhibited inhibitory effect against eighteen (18) bacterial species and four fungi species. Many studies were reported regarding the antimicrobial efficacy of essential oils from peels and peel extracts of Citrus fruits. Madhuri 
et al., (2014) evaluated antimicrobial efficacy of peel extracts of Citrus aurantium and Citrus sinensis against 3 bacteria by agar well diffusion assay. K. pneumoniae exhibited higher susceptibility to peel extracts whereas $B$. cereus was least affected. In our study we observed a 3 $\log$ reduction for $B$. cereus whereas $4 \log$ reduction was shown in case of $K$. pneumoniae. Bacillus cereus being a gram-positive spore forming bacteria the efficacy might be less.

ZITRITIDE exhibited highest antifungal activity against Aspergillus flavus (6 Log Reduction), followed by Aspergillus niger (5 Log reduction). Upon Penicillium species it showed $4 \log$ reductions whereas on $C$. albicans it showed 3 Log reductions. It has been found that the peel extracts of Citrus sinesis significantly inhibited the growth of Fusarium oxysporum to higher extent when compared to leaf extract (OkWu 2007). Citrus aurantium peel extracts exhibited high antifungal activity (>50\%) against Candida capsici as reported by Madhuri et al., (2014).

ZITRITIDE also proved to be effective in combating nosocomial infections. In some critical areas the environment may be heavily contaminated with drug-resistant pathogens like MRSA, Klebsiella pneumoniae, Acinetobacter species and Pseudomonas aeruginosa. As reported by Taneja et al., (2005), 27.3\% of the environmental surfaces of various ICUs and emergency wards showed contamination of Staphylococcus aureus, and 30\% of these were MRSA. In a MRSA outbreak study, Singh et al., (2012) observed the contamination of MRSA in inanimate objects like medicine trolleys, the patient's cabinets, and railing of the beds, the nurses lockers, electric switches and door handles. It was emphasized in several studies that routine cleaning and hand washing alone were not sufficient to control the prolonged outbreaks of MRSA, but proper disinfection was required (Blythe et al., 1998; Rampling et al., 2001). Thus proper disinfection with an effective natural and organic solution is always better than chemical alternatives.
The significant killing efficiency of ZITRITIDE upon MRSA and other pathogens associated with Hospital Acquired Infections proved to be an effective fumigant and a safe alternative to chemical fumigants.

The application of ZITRITIDE in clean room manufacturing facility found to be quite effective as evidenced from the result (Table 9 and 10). The advantage of this fogging solution is that it can be applied in all areas in any time and in a working environment. In this study the fumigation was performed during working hours.

People who are present at the time of spraying, fogging or fumigation, and inhale the micron particles of the disinfectant can clear themselves of all the nasal and throat infections present in them and it will not cause Adina of the lungs. There is no need to shift or move anything or anyone at the time of spraying, fogging or fumigating and the area can be utilized immediately after disinfection. It is an ecofriendly solution and thus safe for people, animals, environment and even children. Thus the beauty of the solution is to apply it easily in all application areas of Hospitals and Industries where chemical fumigants are restricted. It can solve many health problems that arise due to the use of chemical fumigants.

\section{Acknowledgement}

Authors acknowledge the whole technical team involved in the experimental planning and manuscript preparation. Special thanks to Dr. Sarala Devi, Professor and Head, Department of Microbiology, Trivandrum Medical College, Kerala for conducting the fumigation experiment at Hospitals.

\section{References}

Blythe, D., Keenlyside, D., Dawson, S.J., Galloway, A. 1998. Environmental contamination due to Methicillin Resistant Staphylococcus aureus (MRSA). J. Hosp. Infect., 38(1): 67-69. 
(PubMed: 9513070).

Carvalho Freitas, M.I., Costa, M. 2002. Anxiolytic and sedative effects of extracts and essential oil from Citrus aurantium L. Biol. Pharm. Bull., 25(12): 1629-1633.

Damián-Reyna, A.A., González-Hernández, J.C., Carmen Chávez-Parga, Ma. Del. 2015. Current procedures for extraction and purification of citrus flavonoides. Revista Colombiana de Biotechnologia, 18(1): 1-9.

Madhuri, S., Aswini, U.H., Srilakshmi, N.S., T.R., Prashith, K. 2014. Antimicrobial actitvity of Citrus sinensis and Citrus aurantium peel extracts. JPSI, 3(4): 366368.

Ok Wu, De., Awurum, A.N., Okoronkwo, J.I. 2007. Phytochemical composition and in vitro antifungal activity screening of extracts from citrus plants against Fusarium oxysporum of Okra plant (Hibiscus esculentus). African Crop Sci. Proceedings, 8: 1755-1758.

Oliveria, S.A.C., Rabelo, J., Zanbrana, M., Reis Di Iorio, F.B., Periera, A.C., Cardoso Jorge, Cardoso, A.O. 2014. The antimicrobial effects of Citrus limonum and Citrus aurantium essential oils on multispecies biofilms. Braz. Oral. Res. 28(1): 1-6.

Pellati, F., Benvenuti, S., Melegari, M., Firenzouli, F. 2002. Determination of adrenergic agonists from extracts and herbal products of Citrus aurantium L. var. Amara by LC Department of Pharmaceutical Sciences, University of
Modena and Reggio Emilia via Campi 183. Modena Italy. J. Pharm. Biomed. Anal., 29(6): 1113-1119.

Rampling, A., Wiseman, S., Davis, L., Hyett, A.P., Walbridge, A.N., Payne, G.C. et al., 2001. Evidence that hospital hygiene is important in the control of Methicillin Resistant Staphylococcus aureus. 2001. J. Hosp. Infect., 49(2): 109-116.

Singh, M., Sharma, R., Gupta, P.K., Rana, J.K., Sharma, M. and Taneja, N. 2012. Comparative efficacy evaluation of disinfectants routinely used in hospital practice: India. Indian J. Crit. Care Med., 16(3):123-129.

Song, D.K., Suh, H.W., Jung, J.S., Wie, M.B., Son, K.H., Kim, Y.H. 1996. Antidepressant-like effects of $\mathrm{p}$ synephrine in mouse models of immobility tests. Neurosci. Lett., 23(214): 107-110.

Standard Guide for Assessment of Antimicrobial Activity Using a Time-Kill Procedure. ASTM, E 2315 - 16.

Taneja, N., Emmanuel, R., Singh, M., Sharma, M. 2005. Hydrogen peroxide fogging in an overcrowded tertiary care centre: Some practical queries. J. Hosp. Infect., 60(1): 85-92. (PubMed: 15823664).

Taneja, N., Emmanuel, R., Chari, P.S., Sharma, M. 2005. Hospital environment contamination with gram negative bacteria is as common and important as that with gram-positive bacteria in an overloaded tertiary care hospital in India. J. Hosp. Infect., 59(2): 164-165.

\section{How to cite this article:}

Prangya Paramita Tripathy and Oliver Davis George. 2017. Evaluation of Antimicrobial Activity of ZITRITIDE, A Natural and Organic Antimicrobial Fogging Solution with Special Reference for Infection Prevention and Control in Hospital Environments and All Other Clean Room Facilities. Int.J.Curr.Microbiol.App.Sci. 6(4): 1822-1837.

doi: https://doi.org/10.20546/ijcmas.2017.604.218 